\title{
Linx
}

Revue des linguistes de l'université Paris X Nanterre

68-69| 2013

Corpus et apprentissage du français

\section{Comment les données de corpus pourraient renouveler les manuels de grammaire?}

Illustrations à partir de quelques pronoms et de la forme même

Mireille Bilger et Paul Cappeau

\section{OpenEdition}

\section{Journals}

Édition électronique

URL : http://journals.openedition.org/linx/1526

DOI : $10.4000 /$ linx.1526

ISSN : 2118-9692

Éditeur

Presses universitaires de Paris Nanterre

Édition imprimée

Date de publication : 19 novembre 2013

Pagination : 177-199

ISSN : 0246-8743

Référence électronique

Mireille Bilger et Paul Cappeau, "Comment les données de corpus pourraient renouveler les manuels de grammaire? », Linx [En ligne], 68-69 | 2013, mis en ligne le 19 novembre 2015, consulté le 19 avril 2019. URL : http://journals.openedition.org/linx/1526; DOI : 10.4000/linx.1526 


\title{
Comment les données de corpus pourraient renouveler les manuels de grammaire? Illustrations à partir de quelques pronoms et de la forme même
}

\author{
Mireille Bilger, Université de Perpignan Via Domitia, CRESEM \\ Paul Cappeau, Université de Poitiers, FoReLL
}

\section{Introduction}

L'exploitation de grands corpus - de nature et de «genres» différents (oral/écrit, littérature, presse, oral de parole privée, de parole publique, etc.) - a permis de jeter un éclairage neuf sur de nombreux faits de langue et d'en renouveler à la fois l'analyse et la présentation. Ce constat est indéniable notamment pour l'anglais via l'ouvrage de Biber et al. (1999) ou encore, dans le domaine de la lexicographie, le projet Cobuild initié par Sinclair (Sinclair, 1991) ou plus récemment le projet de création de manuels, Touchstone (McCarthy, 2004). En revanche, en ce qui concerne la langue française, ces types de travaux sont encore peu nombreux ou bien trop parcellaires. Pourtant, comme le signalent Arnavielle et Siouffi (2012: 6) :

La réflexion générale autour de la grammaire est active, à l'heure actuelle. Plusieurs colloques, séminaires, journées d'étude, rencontres ont porté, récemment, sur la difficile question de l'adéquation entre outils scientifiques de description et visées (par exemple pédagogiques au sens large) de la grammaire.

ou encore Cadet et Guerin (2012: 4) :

On peut s'interroger sur ce qui est effectivement en jeu dans les différentes didactiques. L'enseignement d'une langue, quelle que soit la perspective (langue 
maternelle, étrangère ou seconde) pose d'emblée le problème de la sélection des formes sur lesquelles repose le savoir transmis : il ne peut s'agir de la langue dans toute l'étendue de son hétérogénéité et de sa dynamique.

Ces citations récentes tirées de deux numéros de revues (Langue Francaise et Le francais aujourd'bui) illustrent bien l'impact que l'exploitation des corpus pourrait avoir aujourd'hui, en France, sur les réflexions touchant à l'enseignement de la grammaire et de la langue maternelle LM ou étrangère LE.

En ce qui concerne les manuels, c'est essentiellement dans le domaine du FLE que l'on trouve des exemples intéressants de choix originaux dans l'approche grammaticale, et certains ouvrages, déjà anciens, tels que Job, Mis et Pissavy (1986), Bérard et Lavenne (1991), De Salins (1996), ou plus récents, comme celui de Lafon et Zeggah-Wuyts (2009), font preuve d'une singularité qui ne trouve pas de réel équivalent dans le domaine du FLM.

Ce décalage s'explique sans aucun doute, comme le montre Vigner (2003), par l'histoire, les traditions et les différentes fonctions que l'on assigne à la langue enseignée qui, de fait, ne sont pas de même nature. Blanche-Benveniste et Jeanjean (1986) ont également montré comment, en voulant répondre aux préoccupations de l'enseignement du FLE (donner une version réaliste de la langue), débutent, dès les années 1950, le recueil de données orales attestées et leur analyse. Au départ, ces dernières sont utilisées pour une meilleure approche du lexique ; par la suite, elles vont timidement être exploitées en vue d'une présentation renouvelée des formes grammaticales (Chambers, 2009).

Ce rapide panorama et ces tendances de fond ne doivent pourtant pas masquer l'essentiel: la grammaire présentée traditionnellement comme celle de la langue est, généralement dans la tradition française, la grammaire d'un usage particulier : la langue écrite littéraire.

La linguistique sur corpus, qui permet à la fois une étude qualitative et quantitative des formes étudiées (Halliday, 1991), révèle que très régulièrement les emplois de telle ou telle forme ne sont pas uniformes mais se distribuent de manière différenciée selon les types et les «genres» de productions (Bilger, 2008; Bilger \& Cappeau, 2004). En ce sens, cette approche méthodologique peut conduire à un réaménagement dans la liste des formes présentées qui tient mieux compte de la fréquence (cf. l'exemple des relatifs composés). Elle peut déplacer la frontière entre faits de lexique et faits de grammaire en montrant que certaines associations de clitiques compléments sont réservées pour l'essentiel à quelques verbes (cf. l'exemple sur l'ordre des pronoms compléments). Elle peut aussi conduire à enrichir le contenu grammatical lui-même en faisant apparaître des structures peu présentes à l'écrit et pour cela ignorées des grammaires alors qu'elles sont fortement présentes à l'oral (cf. l'exemple sur la forme même).

L'objectif de cette contribution est de montrer, à travers ces divers exemples, comment la prise en compte de la diversité des usages permettrait de modifier de 
façon plus ou moins sensible la description de certains faits de langue et au-delà la représentation de la grammaire dans les manuels (Conrad, 2000).

\section{La situation des corpus oraux en France}

La situation des corpus en France est quelque peu singulière et atypique. L'élaboration de Frantext (dans les années 1970) constituait une avancée majeure et plutôt novatrice dont la portée a toutefois été fortement limitée par deux facteurs : l'orientation littéraire de plus de $80 \%$ des documents rassemblés et la non prise en compte de documents oraux. Des tentatives originales très anciennes ont pourtant, à différentes périodes, été amorcées : les Archives de la Parole (dans les années 1910), le français fondamental (années 1950), le corpus d'Orléans (années 1970), le corpus du GARS (à partir des années 1980). Il existait donc à côté de l'ambitieux projet sur l'écrit un matériau oral (ainsi qu'une réflexion et un savoir-faire) qui a été quelque peu négligé. C'est probablement de ce rendez-vous manqué avec la langue parlée (et de son corollaire : une surreprésentation de l'écrit littéraire) que l'on peut dater l'important retard pris par la France dans la constitution de corpus représentatifs de la langue en usage. Retard qui n'a pas touché aussi fortement d'autres langues européennes telles que l'anglais, l'italien, le portugais ou l'espagnol, car l'emprise de la langue écrite littéraire était certainement moins marquée.

Il faut dès lors attendre le début du 21 e siècle pour que plusieurs projets donnent accès de façon simplifiée à des données orales et à des transcriptions. Depuis les années 2000, le paysage est, de fait, en pleine transformation et plusieurs grands projets, élaborés dans des perspectives différentes, ont émergé et continuent à se développer. On peut citer notamment ${ }^{1}$ :

- ESLO (Enquêtes sociolinguistiques à Orléans) qui a nettoyé le célèbre corpus d'Orléans (ESLO 1) et a prolongé ce travail (ESLO 2, Baude \& Dugua, 2011);

- PFC (Phonologie du français contemporain) qui a développé des enquêtes (lectures, entretiens) à travers le territoire français et l'a élargi à la francophonie (Detey et al., 2010) ;

- CFPP2000 (Corpus de français parlé parisien des années 2000) qui est constitué d'entretiens longs avec des locuteurs parisiens (Branca-Rosoff et al., 2011) ;

- CRFP (Corpus de référence du français parlé) - dont on attend toujours la disponibilité - collecte de situations (privée, publique et professionnelle) dans les grandes villes de l'hexagone (Equipe DELIC, 2004);

- CIEL-F (Corpus d'interactions écologiques de la langue française) constitué de situations non provoquées, recueillies dans la francophonie (Gadet et al., 2009).

Ce rapide panorama montre qu'il est aujourd'hui envisageable, pour un large public de spécialistes mais aussi d'utilisateurs avertis, de disposer d'échantillons larges d'oral attesté, produit dans des situations variées, et qu'il est également possible de

${ }^{1}$ Un inventaire détaillé des corpus oraux figure sur le site de l'IRCOM (http://ircom.corpusir.fr/site/corpus.php). Voir aussi Delahaie, ce volume. 
récupérer des exemples (transcription + son) de multiples structures ou de valider des intuitions sur certains usages (comme le montreront les paragraphes suivants).

\section{La présentation des relatifs composés}

S'il est un secteur où les choix de présentation effectués dans les grammaires et les manuels sont peu convaincants, c'est bien celui de l'ordre de succession des relatifs. Pour les formes simples, c'est l'ordre latin qui, depuis des siècles, est repris... Ce qui ne peut manquer de surprendre. En ce qui concerne les formes composées, il est plus difficile de déterminer ce qui guide l'organisation.

Ainsi, Abry et Chalaron (2000) dans la Grammaire des premiers temps proposent un tableau qui recense les associations entre des prépositions (fournies dans la première colonne) et les formes en lequel. L'organisation de ce tableau (Tableau 1) semble obéir à deux logiques distinctes :

\begin{tabular}{|c|c|}
\hline Prépositions & \multicolumn{1}{c|}{ lequel, laquelle, lesquels, lesquelles } \\
\hline après & \\
\hline avec & \\
\hline chez & \\
\hline contre & \\
\hline$[\ldots]$ & \\
\hline selon & \\
\hline sur & \\
\hline à & \\
\hline grâce à & \\
\hline face à & \\
\hline au cours de & \\
\hline au-delà de & \\
\hline au milieu de & \\
\hline auprès de & \\
\hline
\end{tabular}

Tableau 1 :

Extrait de la présentation des relatifs composés (Grammaire des premiers temps, 2000)

Dans une première partie de la liste (de après jusqu'à sur), c'est l'ordre alphabétique qui est adopté. Ce choix, s'il peut répondre à un souci pratique - la facilité pour retrouver une forme - reste néanmoins atypique (car les faits de grammaire répondent rarement à une logique alphabétique) et n'est peut-être pas le plus indiqué quand la liste comporte peu d'unités. Ce mode de présentation fournit en tout cas peu d'indications sur l'emploi grammatical (les propriétés, les conditions d'emploi, la fréquence des assemblages, etc.).

La deuxième partie de la liste - qui n'est pas formellement séparée de la première - vient brouiller l'apparente logique d'organisation du début puisque la 
succession repart de à et contient des locutions prépositives qui ne respectent pas l'ordre alphabétique.

L'observation de deux corpus diversifiés ${ }^{2}$ - afin de ne pas proposer un seul usage de la langue aux apprenants - fournit les résultats globaux présentés dans le Tableau 2. Ces résultats mettent à mal le tableau précédent sur plusieurs points et permettent de dégager un certain nombre d'observations, qui confortent et parfois complètent les observations de Bilger (2008) :

\begin{tabular}{|l|c|c|c|c|c|c|c|c|c|c|c|}
\hline & \multicolumn{3}{|c|}{ ECRIT } & TOT & \multicolumn{5}{c|}{ ORAL } & TOT & Rang \\
\hline & lequel & laquelle & lesquels & lesquelles & & lequel & laquelle & lesquels & lesquelles & & \\
\hline dans & 42 & 42 & 20 & 9 & $\mathbf{1 1 3}$ & 42 & 20 & 7 & 5 & $\mathbf{7 4}$ & $\mathbf{2}$ \\
\hline sur & 47 & 26 & 15 & 7 & $\mathbf{9 5}$ & 18 & 0 & 4 & 11 & $\mathbf{3 3}$ & $\mathbf{4}$ \\
\hline avec & 26 & 37 & 7 & 3 & $\mathbf{7 3}$ & 9 & 3 & 6 & 2 & $\mathbf{2 0}$ & $\mathbf{5}$ \\
\hline pour & 10 & 8 & 5 & 7 & $\mathbf{3 0}$ & 5 & 11 & 7 & 10 & $\mathbf{3 3}$ & $\mathbf{7}$ \\
\hline contre & 2 & 3 & 3 & 3 & $\mathbf{1 1}$ & 1 & 1 & 0 & 0 & $\mathbf{2}$ & $\mathbf{1 3}$ \\
\hline devant & 5 & 8 & 2 & 1 & $\mathbf{1 6}$ & 1 & 1 & 1 & 1 & $\mathbf{4}$ & $\mathbf{8}$ \\
\hline par & 25 & 20 & 14 & 5 & $\mathbf{6 4}$ & 1 & 1 & 0 & 1 & $\mathbf{3}$ & $\mathbf{6}$ \\
\hline pendant & 2 & 6 & 3 & 4 & $\mathbf{1 5}$ & 1 & 1 & 0 & 0 & $\mathbf{2}$ & $\mathbf{9}$ \\
\hline sous & 7 & 4 & 1 & 0 & $\mathbf{1 2}$ & 1 & 1 & 0 & 0 & $\mathbf{2}$ & $\mathbf{1 2}$ \\
\hline à & 106 & 85 & 38 & 32 & $\mathbf{2 6 1}$ & 20 & 19 & 9 & 6 & $\mathbf{5 4}$ & $\mathbf{1}$ \\
\hline$(\ldots)$ de & 45 & 57 & 24 & 16 & $\mathbf{1 4 2}$ & 3 & 6 & 1 & 0 & $\mathbf{1 0}$ & $\mathbf{3}$ \\
\hline selon & 0 & 0 & 0 & 0 & $\mathbf{0}$ & 0 & 1 & 0 & 0 & $\mathbf{1}$ & $\mathbf{2 0}$ \\
\hline vers & 4 & 7 & 1 & 0 & $\mathbf{1 2}$ & 0 & 1 & 0 & 0 & $\mathbf{1}$ & $\mathbf{1 3}$ \\
\hline parmi & 0 & 0 & 6 & 3 & $\mathbf{9}$ & 0 & 0 & 6 & 1 & $\mathbf{7}$ & $\mathbf{1 0}$ \\
\hline chez & 6 & 3 & 3 & 3 & $\mathbf{1 5}$ & 0 & 0 & 1 & 0 & $\mathbf{1}$ & $\mathbf{1 0}$ \\
\hline derrière & 4 & 2 & 2 & 3 & $\mathbf{1 1}$ & 0 & 0 & 0 & 0 & $\mathbf{0}$ & $\mathbf{1 5}$ \\
\hline entre & 0 & 0 & 2 & 1 & $\mathbf{3}$ & 0 & 0 & 0 & 0 & $\mathbf{0}$ & $\mathbf{1 9}$ \\
\hline sans & 0 & 5 & 1 & 0 & $\mathbf{6}$ & 0 & 0 & 0 & 0 & $\mathbf{0}$ & $\mathbf{1 7}$ \\
\hline après & 3 & 1 & 0 & 0 & $\mathbf{4}$ & 0 & 0 & 0 & 0 & $\mathbf{0}$ & $\mathbf{1 8}$ \\
\hline $\begin{array}{l}\text { par- } \\
\text { dessus }\end{array}$ & 1 & 0 & 0 & 0 & $\mathbf{1}$ & 0 & 0 & 0 & 0 & $\mathbf{0}$ & $\mathbf{1 9}$ \\
\hline $\begin{array}{l}\text { à } \\
\text { travers }\end{array}$ & 1 & 5 & 3 & 0 & $\mathbf{9}$ & 0 & 0 & 0 & 0 & $\mathbf{0}$ & $\mathbf{1 6}$ \\
\hline & & & $\mathbf{9 0 2}$ & & & & & $\mathbf{2 4 7}$ & & & \\
\hline
\end{tabular}

Tableau 2 : Distribution des prépositions dans deux corpus de 1 million de mots

Le phénomène observé relève bien plus de la langue écrite que de l'oral puisque les exemples recueillis sont trois fois plus nombreux dans le corpus écrit. D'autre part,

2 Chaque corpus comprend 1 million de mots. Le corpus oral est constitué de transcriptions de thématiques différentes établies à Clermont-Ferrand, Nancy, Grenoble et Poitiers, cela afin de disposer d'un échantillon de l'usage courant. Le corpus écrit est constitué de textes littéraires $19 \mathrm{e}$ siècle pour l'essentiel, dans le souci d'observer une langue proche de l'usage décrit par les grammaires. 
la diversité des prépositions y est aussi plus importante (les six dernières prépositions du tableau n'ont pas été trouvées dans le corpus oral) ${ }^{3}$.

Le choix des prépositions simples retenues dans le tableau du manuel surprend quelque peu puisque certaines des formes présentées (durant, par-dessous) n'ont pas été observées dans nos productions ; inversement, des prépositions qui sont attestées en nombre (devant, pendant, etc. - voir les exemples 1 et 2 ) ne sont pas fournies dans le document proposé aux apprenants.

1. Après environ une demi-heure, pendant laquelle mademoiselle de Verneuil et d'Orgemont se regardèrent [...] (Ecrit, Balzac)

2. en face de cette situation devant laquelle euh il se trouvait tout à coup de devoir répondre à cette question (Oral, P98)

L'arbitraire du choix, c'est du moins ainsi que l'on interprétera ces faits, ne peut que faire ressortir l'intérêt des corpus qui «encadrent» ou «orientent» les données qu'il est utile et pertinent de proposer à des apprenants.

Si l'on s'en tient aux seules données orales, une hiérarchie se dégage très fortement et réduit drastiquement les formes qui méritent d'être présentées. Seules quatre prépositions semblent réellement productives : dans, sur, pour, avec. On peut aussi ajouter $\grave{a}$ (et dans une bien moindre mesure $d e$ ) qui intervient dans la fabrication de formes synthétiques (anquel, auxquelles...).

L'appui sur des données de corpus déplace ainsi le travail grammatical qui de l'exploitation d'une mécanique quelque peu artificielle de la combinaison avec une préposition (piochée dans une liste très vaste) s'oriente vers la maitrise d'une construction en relation avec des usages attestés.

\section{L'ordre des pronoms compléments}

Les règles d'apparition des pronoms compléments quand ils sont cumulés comme dans (3)-(4) font partie des faits de langue qui trouvent place dans la plupart des grammaires.

3. Pierre le lui donne

4. Marie lui en a parlé

Les explications proposées dans les ouvrages (grammaires et manuels) relèvent de deux types distincts. Les unes, plus anciennes, prennent appui sur les fonctions que remplissent ces pronoms. Elles donnent lieu à des schémas souvent complexes qui

\footnotetext{
${ }^{3}$ Bilger (2008) identifie à partir d'autres corpus écrits (un corpus de presse notamment) certaines collocations non observées dans le corpus littéraire. On peut citer en particulier: au cours de laquelle/ duquel ou selon lequel / laquelle et les assemblages tels que condition(s) dans laquelle / lesquelles et raison(s) pour laquelle / lesquelles qui peuvent être vus comme des clichés de l'écriture journalistique. Inversement la préposition avec était peu observée.
} 
peuvent même apparaitre comme confus. On en trouve par exemple dans Bonnard (1981) ou dans Arrivé et al. (1986), d'où est extrait le document ci-après (Figure 1) :

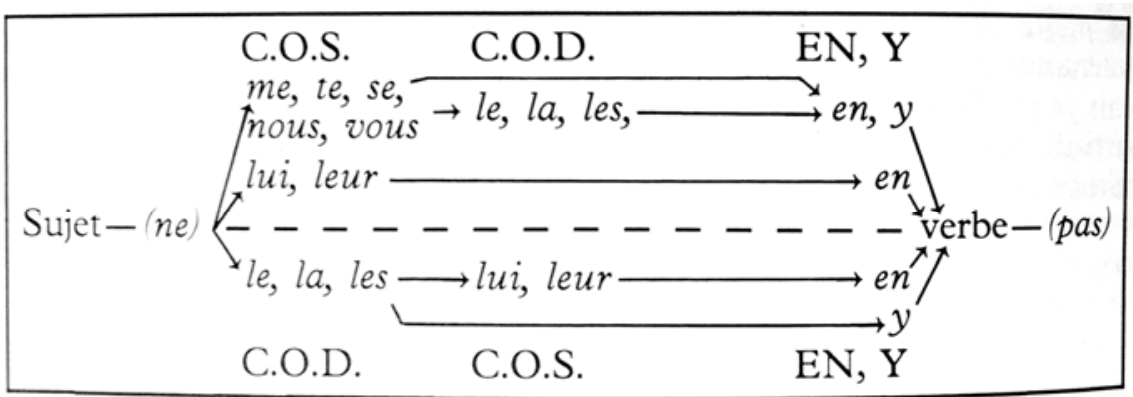

Figure 1 : L'ordre des pronoms compléments dans Arrivé, Gadet \&Galmiche (1986)

Les secondes s'appuient sur les séries des formes elles-mêmes (c'est-à-dire les paradigmes de type le, la, les vs lui, leur). Cette orientation s'est progressivement substituée à l'approche précédente. On la trouve par exemple dans McBride (1997) et dans divers manuels comme Grammaire pratique du Fransais (2000). Voici le résumé partiel de la combinatoire que propose ce dernier ouvrage (Figure 2) :

\section{Quand il y a deux pronoms compléments}

Avec " en "

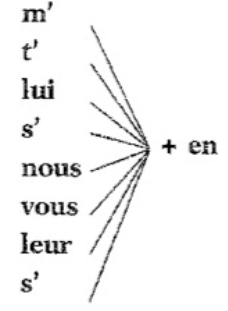

Avec "le, la, les"

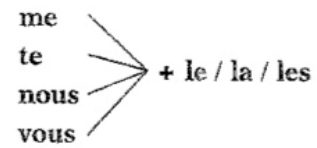

Avec " lui, leur"

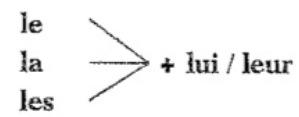

Elle me donne un livre.

$\rightarrow$ Elle m'en donne un.

On lui a offert des rollers.

$\rightarrow$ On lui en a offert.

Elle nous donnera son livre.

$\rightarrow$ Elle nous le donnera.

Il m'a prêté sa moto.

$\rightarrow$ Il me l'a prêtée.

I enverra sa nouvelle adresse à ses amis.

$\rightarrow$ Il la leur enverra.

Figure 2. L'ordre des pronoms compléments dans Grammaire pratique du français.

D'un point de vue grammatical, l'exhaustivité qu'apporte cette présentation peut sembler, à première vue, satisfaisante et parfaitement adaptée aux besoins des apprenants. Toutes les suites potentielles sont indiquées et mises à disposition du lecteur. 
Une autre vision du problème de l'ordre des pronoms compléments peut être fournie par un ouvrage comme celui de Blanche-Benveniste (2010) qui élabore sa description à partir des distributions observées dans des corpus. Plusieurs points en ressortent et conduisent à adopter un regard plus critique sur la présentation précédente. Premièrement, il existe un fort déséquilibre entre les diverses séquences virtuelles de deux clitiques. Seules quelques-unes sont réellement exploitées par les locuteurs : $y+e n, s e+e n$, me $+e n$. D'autres suites, souvent mises en avant dans les grammaires ou les manuels comme le + lui ou le + leur, sont quasi exceptionnelles. Ainsi les constructions avec un réfléchi sont privilégiées par les usagers mais pas nécessairement par l'enseignement.

Deuxièmement, les données de corpus sont éclairantes sur la vision quelque peu déformée propagée par les grammaires. Le Tableau 3 permet de vérifier que le nombre d'occurrences des suites le lui et s'en est très déséquilibré (en faveur de s'en, ce que les grammaires ne signalent pas) et que ces suites sont massivement utilisées à l'écrit mais peu à l'oral (en particulier pour le luı):

\begin{tabular}{|c|c|c|}
\hline & le lui & s'en \\
\hline Ecrit (Litt 19e) ${ }^{4}$ & 57 & 525 \\
\hline Oral (divers) & 7 & 115 \\
\hline
\end{tabular}

Tableau 3 : Nombre d'occurrences des suites de clitiques

En outre, pour chaque suite, un verbe tient une place prépondérante. Ainsi à l'écrit, la suite le lui se rencontre dans $28 \%$ des cas avec le verbe dire (5) et s'en dans $35 \%$ des cas avec le verbe aller (6) :

5. Je lui ai pardonné, vous pouvez le lui dire. (Ecrit, Zola)

6. Et elle s'en alla, précédée du vendeur [...] (Ecrit, Zola)

Troisièmement, Blanche-Benveniste (2010) a proposé à divers locuteurs des suites de clitiques. Il en ressort que certaines suites (comme le lui en particulier) sont acceptées plus facilement avec quelques verbes particuliers comme donner, prêter et faire et que les locuteurs sont plus réticents face à ces assemblages avec d'autres verbes comme envoyer ou emprunter. Cela montre qu'une présentation mécanique de ce fait grammatical (qui ne tient pas compte des séquences de clitiques et des verbes impliqués) ne peut convenir.

Ces rapides observations aboutissent à s'interroger sur la frontière entre lexique et syntaxe que les corpus incitent à reconsidérer (Sinclair, 1991). Un point, comme la suite de deux clitiques compléments, habituellement traité en grammaire (d’où le souci de l'exhaustivité dans les combinaisons présentées) semble plutôt relever d'un fait de lexique (ce sont quelques combinaisons clitiques + verbes qui méritent d'être apprises et retenues). La présentation dans les manuels pourrait tirer profit de ces informations

${ }^{4}$ Il s'agit des corpus présentés plus haut. 
pour ne présenter qu'un nombre très limité de combinaisons et renvoyer à des annexes les tableaux des combinaisons potentielles si peu attestées.

\section{La description de même}

Les grammaires de FLM fournissent assez peu d'indications unifiées sur de nombreux morphèmes, ce qui est effectivement le cas pour la forme même. Le fait que cette dernière appartienne à plusieurs catégories et possède divers sémantismes favorise sans aucun doute l'éparpillement de l'analyse.

Ainsi, l'index de la nouvelle édition de la Grammaire méthodique du français (Riegel et al., 2009) renvoie le lecteur à de multiples paragraphes et le conduit à consulter 10 pages différentes pour connaitre les divers emplois de la forme même. C'est donc une image éclatée de cet élément qui semble s’imposer.

En ce qui concerne les ouvrages de FLE, la forme même est rarement mentionnée - si ce n'est dans La nouvelle grammaire du franscais (Delatour et al., 2004) - et quand elle l'est, c'est dans des emplois qui se révèlent marginaux ${ }^{5}$ (cf. par exemple, quand bien même dans La grammaire des premiers temps (Abry et al., 1999)), alors que, comme nous le verrons, cette forme est bien représentée dans les productions, aussi bien orales qu'écrites. Elle occupe d'ailleurs la $53^{\text {e }}$ place dans la liste des 200 mots les plus utilisés en français parlé (cf. l'inventaire fourni par Blanche-Benveniste, 2010), d'où l'intérêt de s'appuyer sur des corpus pour en mieux cerner les emplois.

Si les descriptions disponibles s'appuient sur des catégories bien installées (adverbe, pronom et adjectif) et bifurquent rapidement vers des commentaires sémantiques ${ }^{6}$, les données de corpus vont nous permettre de fournir de nouvelles pistes d'analyse et de proposer une description à la fois plus homogène et plus détaillée.

\subsection{Rapide présentation du cadre d'analyse}

\subsubsection{Les corpus utilisés}

Quatre corpus de taille identique (200 000 mots chacun) mais de nature différente ont été utilisés pour mener à bien cette étude :

- le corpus oral Parole Privée (oral Privé) est constitué d'une partie du Corpus de Référence de Français Parlé (DELIC, 2004);

- le corpus oral Parole Publique/Professionnelle (oral Pro/oral Public) est également constitué d'une partie du CRFP à laquelle s'ajoutent des corpus $^{7}$ de paroles professionnelles (avocats, hommes politiques, etc.);

\footnotetext{
${ }^{5}$ En tout cas du point de vue quantitatif (voir plus loin).

${ }^{6}$ Le TLF, par exemple, propose un classement qui entrecroise deux types de classement: une première partie repose sur la valeur sémantique alors qu'une deuxième se fonde sur la position.

${ }^{7}$ Ces corpus complémentaires ont été édités par Paul Cappeau.
} 
- le corpus Littérature (Litt) comprend des œuvres romanesques classiques du XIX siècle (entre autres, Zola, Balzac, Hugo, Flaubert) ;

- le corpus de Presse (Presse) est constitué d'articles de certains quotidiens et hebdomadaires (Le Monde, Libération ou Le Nouvel Observateur).

Les résultats bruts obtenus figurent dans le Tableau 4 :

\begin{tabular}{|l|c|c|c|c|}
\hline \multirow{2}{*}{} & \multicolumn{2}{|c|}{ ORAL } & \multicolumn{2}{c|}{ ECRIT } \\
\cline { 2 - 5 } & Privé & Public / Pro & Littéraire & Presse \\
\hline $\begin{array}{l}\text { Nombre } \\
\text { occurrences }\end{array}$ & 802 & 885 & 440 & 393 \\
\hline
\end{tabular}

Tableau 4 : Les occurrences de même(s)

Dans les corpus oraux, la forme même est deux fois plus employée; nous verrons par la suite que ce phénomène est étroitement lié à la forte proportion de la locution quand même.

\subsubsection{Paramètres d'analyse}

Pour rendre compte du fonctionnement de même, nous avons eu recours à divers paramètres :

- la variation morphologique, la distribution (c'est-à-dire la position de même et la nature du groupe dans lequel il prend place) et la sémantique ;

- le travail sur corpus nous a également permis de dégager un certain nombre de collocations et d'observer leur inégale répartition selon les corpus utilisés.

La morphologie constitue le premier critère qui permet de subdiviser en deux l'ensemble des formes retenues, selon la répartition suivante (Tableau 5) :

\begin{tabular}{|l|l|}
\hline \multicolumn{1}{|c|}{ même forme variable } & \multicolumn{1}{c|}{ même forme invariable } \\
\hline - vous aurez les mêmes produits (oral Pub) & - des gens dangereux ou même méprisables \\
- on voit toujours les mêmes (Litt) & (Litt) \\
& $\begin{array}{l}\text { - ils ont } \text { même une très grande marque de } \\
\text { bière (oral Pri) }\end{array}$ \\
\hline
\end{tabular}

\section{Tableau 5 : Classement des deux formes de même}

La colonne de droite correspond à ce que l'on appelle traditionnellement l'emploi adverbial, qui sera détaillé dans le 5.3.

La colonne de gauche regroupe à la fois ce que l'on désigne comme l'emploi adjectival et pronominal. La distribution dans les corpus est alors la suivante (Tableau 6) :

\begin{tabular}{|l|c|c|c|c|}
\hline \multirow{2}{*}{} & \multicolumn{2}{|c|}{ ORAL } & \multicolumn{2}{c|}{ ECRIT } \\
\cline { 2 - 5 } & Privé & Public / Pro & Littéraire & Presse \\
\hline même variable & $174(21,6 \%)$ & $321(36 \%)$ & $249(56,60 \%)$ & $188(48 \%)$ \\
\hline même invariable & $628(78,4 \%)$ & $564(64 \%)$ & $191(43,40 \%)$ & $206(52 \%)$ \\
\hline
\end{tabular}

Tableau 6 : Répartition des deux formes dans les différents corpus 
Ce simple relevé esquisse déjà une certaine singularité du corpus littéraire, puisque c'est le seul qui présente la forme variable comme majoritaire. En ce sens, il n'est pas surprenant que les dictionnaires et en particulier le TLF qui s'appuie sur Frantext (composé à $80 \%$ de textes littéraires) commencent régulièrement leur article sur même par la description de l'adjectif.

Notre analyse portera d'abord sur la forme variable (plus rapide à décrire) puis sur la forme invariable qui oblige à regarder diverses locutions.

\subsection{Même : forme variable}

Dans ce cas, il est nécessaire de tenir compte de la position qu’occupe même.

\subsubsection{Même postposé}

Postposée, cette forme variable se rencontre principalement dans 2 contextes : soit après un pronom personnel disjoint, soit après un nom. La réalisation de même après un pronom tonique s'effectue différemment selon les personnes et les types de corpus. L'écrit littéraire présente le total le plus élevé (3 fois plus d'occurrences que dans l'oral privé) par l'emploi massif de lui/elle-même, alors que dans l'oral privé, sans surprise, la forme moi-même se rencontre fréquemment :

7. Fontanet lui-même en fut étonné (écrit Litt)

8. puis j’ai ensuite moi-même fait l'école hôtelière à Toulouse (oral Pro)

La forme même peut être réalisée après le nom tête du groupe nominal :

9. il faut que ça fasse effet le jour même (oral Privé)

10. c'était dès cette nuit même qu'il fallait ...(écrit Litt)

11. les processus mêmes du développement se sont ralentis (Presse)

Ces emplois postposés peuvent être rapprochés des précédents et, comme nous pouvons le voir (Tableau 7), ils sont nettement plus utilisés dans l'écrit littéraire.

\begin{tabular}{|c|c|c|c|c|}
\hline \multirow{2}{*}{} & \multicolumn{2}{|c|}{ ORAL } & \multicolumn{2}{c|}{ ECRIT } \\
\cline { 2 - 5 } & Privé & Public / Pro & Littéraire & Presse \\
\hline Nom + même & 13 & 11 & $56(22 \%)$ & 11 \\
\hline
\end{tabular}

Tableau 7 : Les emplois de même postposé à un nom

Quand il précède le nom (voir 5.2.2), même marque l'identité ou mieux la similitude. Quand il est placé après il est censé indiquer que le nom possède une qualité par excellence, mais les noms attestés dans les corpus ne rendent pas cette notion de qualité très explicite ${ }^{8}$ :

\footnotetext{
${ }^{8}$ Vaugelas semble analyser même comme adverbe dans cette configuration. Il propose d'ailleurs de désaccorder le nom et l'adverbe même pour souligner sa valeur : les choses même que je vous ai dites vs la chose mêmes que je vous ai dite. La surprise vient de Brunot et Bruneau (1956) qui semblent valider cette analyse. Damourette et Pichon consacrent un paragraphe à cette question (\$ 2894) «les auteurs se sont souvent comportés d'une façon assez capricieuse quant à la variation dans ce cas ». Pour eux, les
} 
12. il faut que ça fasse effet le même jour

13. il faut que ça fasse effet le jour même (oral Privé)

\subsubsection{Même antéposé}

L'emploi, de loin le plus massif, excepté dans le corpus Littéraire, est celui dans lequel même est placé avant le nom, le plus souvent entre le déterminant et le nom :

14. as-tu l'impression d'avoir les mêmes relations avec tous les clients (oral Pro)

15. vous savez quand on est deux pour défendre un même client (oral Pro)

On observe une très forte prédominance de l'article défini dans ce contexte (cf. Tableau 8) et des collocations différentes selon les productions. Dans les productions orales, un tiers des occurrences de la suite la même $+\mathrm{N}$ se réalisent sous la forme la même chose. Dans l'écrit de la Presse, pour la séquence $d u$ même $+\mathrm{N}$, on trouve $d u$ même coup dans 40\% des emplois et les séquences au même $+\mathrm{N}$ utilisent pour $60 \%$ des cas, deux lexèmes particuliers : moment et titre (au même moment/ au même titre) :

\begin{tabular}{|l|c|c|c|c|}
\hline \multirow{2}{*}{} & \multicolumn{2}{|c|}{ ORAL } & \multicolumn{2}{c|}{ ECRIT } \\
\cline { 2 - 5 } & Privé (103) & $\begin{array}{c}\text { Public / Pro } \\
(185)\end{array}$ & Littéraire (67) & Presse (106) \\
\hline article défini & 98 & 171 & 56 & 96 \\
\hline article indéfini & 2 & 10 & 9 & 9 \\
\hline démonstratif & 3 & 4 & 2 & 1 \\
\hline
\end{tabular}

Tableau 8 : Les déterminants utilisés devant même + Nom

Ce que l'on désigne traditionnellement comme pronom indéfini (le même et ses variantes) peut être traité comme un cas particulier de la structure plus générale le même $[+\mathrm{N}]:$

16. dans une cave la famille de fromages est la même (oral Pro)

17. parce que l'objet n'est plus le même (oral Privé)

Le Tableau 9 fournit la faible distribution de cet emploi dans les corpus :

\begin{tabular}{|l|c|c|c|c|}
\hline \multirow{2}{*}{} & \multicolumn{2}{|c|}{ ORAL } & \multicolumn{2}{c|}{ ECRIT } \\
\cline { 2 - 5 } & Privé & Public / Pro & Littéraire & Presse \\
\hline proneme & $\mathbf{1 2}$ & $\mathbf{3 3}$ & $\mathbf{5}$ & $\mathbf{9}$ \\
\hline la même & & & 1 & 2 \\
\hline le même & 4 & 10 & 1 & 3 \\
\hline les mêmes & 3 & 16 & 3 & 4 \\
\hline
\end{tabular}

Tableau 9 : Les emplois de même « pronom »

deux emplois (adverbe / adjectif) sont possibles dans ce contexte (les consuls même viendront et les consuls mêmes viendront) un critère permet la distinction : le caractère déplaçable de même lorsqu'il est adverbe. 
Cette rubrique "pronom» apparaît toutefois avoir un intérêt réduit dans la description et son rapprochement avec l'« adjectif» semble nettement plus pertinent 9 De fait, il semble bien que ce statut de forme pronominale pose problème et mérite qu'on s'y attarde un peu. Parmi les particularités de cette forme, on peut rappeler que : premièrement, même doit être accompagné nécessairement d'un article défini :

18a. les enduits sont toujours les mêmes (oral Pro)

18b. * les enduits sont toujours mêmes / des mêmes

Deuxièmement, l'interprétation de ce groupe nécessite un calcul anaphorique qui permet de récupérer un antécédent. Flaux (2008) qui classe le même dans les pronoms précise que «son fonctionnement anaphorique est dû au prédicat bivalent même qui suppose nécessairement la mention préalable d'un $\mathrm{N}$ désignant un référent ». Contrairement à d'autres formes comme certains ou beaucoup (19), aucun emploi nominal avec le trait /+ humain/ n'est attesté en l'absence du référent :

19a. on craint des risques de plans sociaux massifs et certains parlent d'un troisième tour social $\left(\mathrm{O}-\mathrm{HP} 3^{10}\right)$

19b. je crois que beaucoup disaient que et m'ont fait savoir et pas seulement à gauche m'ont fait savoir que s'ils avaient pu signer (O-HP3)

En ce sens, un exemple comme (20) ne peut s'interpréter que si l'antécédent humain a été donné antérieurement :

20. je crois que les mêmes disaient qu'ils n'auraient pas signé

Troisièmement, l'ensemble le même et variantes rappelle le fonctionnement qu'on trouve dans des séquences comme le rouge, le dernier (21), pour lesquelles on peut parler de syntagmes nominaux sans lexème nominal (cf. Blanche-Benveniste, 1990, 2010) :

21a. Cette nuit-là, Simon rêva qu'on pouvait reconnaître les couleurs par le toucher. Le rouge était proche de la liquidité, une consistance un peu sirupeuse. (Frantext, Beck, p. 25)

21b. J'ai viré les petits gars d'une pichenette et le dernier a poussé un hurlement terrible en basculant dans le vide, comme si je lui avais arraché un bras. (Frantext, Djian, p. 137)

Dans les séquences «le/la/les même(s) », le lexème n'est pas réalisé et même est antéposé à ce lexique non réalisé :

21c. il a acheté une voiture

j’ai acheté la même voiture

j’ai acheté la même 0

\footnotetext{
${ }^{9}$ Il est d'ailleurs intéressant de noter que dans les grammaires (cf. entre autres Grevisse, 1980), dans le chapitre concernant les pronoms indéfinis, si même est régulièrement signalé, il ne donne pas pour autant lieu à une entrée particulière comme c'est le cas pour certains, quelqu'un, etc., et la plupart des grammaires FLE ne le signalent pas dans la liste des pronoms indéfinis. De même, certains dictionnaires (TLF) présentent même essentiellement comme étant un adjectif ou un adverbe, tout en signalant que les séquences «le/ la/ les même(s)» sont des groupes pronominaux.

${ }^{10} \mathrm{O}-\mathrm{HP} 3$ est un corpus d'interviews d'hommes politiques.
} 
Dans cet emploi où la forme même est antéposée au lexème (réalisé ou pas), celle-ci marque l'identité ou la similitude selon les contextes :

22. ils avaient droit à une fouille à corps tous les jours fouille à corps ensemble dans une même cellule (oral Pro)

23. est-ce que pour les mêmes produits vous aurez les mêmes produits de vente dans vos différents établissements (oral Pro)

24. mon vote sera le même (oral Pro)

On peut donc s'étonner de l'étiquette classique qui traite le même comme étant un pronom indéfini. En effet, comme on vient de le rappeler, le contexte antérieur ne peut être rempli que par l'article défini, le fonctionnement souligné par Flaux (2008) insiste sur le lien anaphorique obligatoire avec un SN exprimé, enfin le sens de même peut difficilement renvoyer à une valeur de «mal défini».

Le Tableau 10 présente une synthèse concernant la distribution de l'emploi variable de même dans les divers corpus et les collocations observées :

\begin{tabular}{|c|c|}
\hline \multicolumn{2}{|c|}{ même forme variable } \\
\hline même postposé & même antéposé \\
\hline $\begin{array}{l}\text { - à un pronom } \\
\text { - lui dans l'écrit littéraire (lui-même) } \\
\text { - moi dans l'oral privé (moi-même) }\end{array}$ & $\begin{array}{l}\text { - à un nom } \\
\text { - chose est le nom le plus utilisé à l'oral : même } \\
\text { chose } \\
\text { - fréquent dans la presse : du même coup, au même } \\
\text { moment, au même titre }\end{array}$ \\
\hline $\begin{array}{l}\text { - à un nom } \\
\text { - surtout dans l'écrit littéraire (cette nuit même) }\end{array}$ & $\begin{array}{l}\text { - à un nom vide, non réalisé } \\
\text { - emploi le plus fréquent dans l'oral } \\
\text { professionnel (ce sont les mêmes) }\end{array}$ \\
\hline $\begin{array}{l}->\text { marque le caractère en propre du terme } \\
\text { antérieur }\end{array}$ & $\begin{array}{l}\text {-> même possède une valeur assez affaiblie, il } \\
\text { pourrait souvent être remplacé par un } \\
\text { démonstratif : au même moment / à ce moment, au } \\
\text { même titre / à ce titre }\end{array}$ \\
\hline
\end{tabular}

Tableau 10 : Synthèse des emplois de même variable

Le corpus Littéraire se caractérise par un emploi massif de la forme même variable et une répartition assez équilibrée entre 3 emplois : postposé au pronom et au nom et antéposé au nom. Les corpus oraux se caractérisent par un emploi minoritaire de la forme variable et par le fait que les deux tiers des emplois sont antéposés au nom. Le corpus Presse se situe entre les deux autres types de corpus : proche du Littéraire puisque la forme variable est bien représentée, mais aussi proche de l'oral par l'emploi massif de même antéposé au nom.

\subsection{Même : forme invariable}

Le simple décompte des occurrences de même dans des corpus de taille comparable (cf. le tableau 4) pourrait laisser penser que cette forme se rencontre bien plus fréquemment à l'oral qu'à l'écrit (environ deux fois plus). Les tendances globales 
repérées dans les différents corpus sont en effet très convergentes et semblent neutraliser la distinction selon les types de production (privé, public, littéraire, presse) au profit de la seule différence entre oral et écrit.

Cependant, cette première impression est grandement faussée par le poids de la locution quand même qui représente de 30 à $45 \%$ des emplois à l'oral contre moins de 2 $\%$ à l'écrit. Si l'on écarte cette locution de l'inventaire, les données chiffrées apparaissent bien plus équilibrées (Tableau 11) :

\begin{tabular}{|l|c|c|c|c|}
\hline \multirow{2}{*}{} & \multicolumn{2}{|c|}{ ORAL } & \multicolumn{2}{c|}{ ECRIT } \\
\cline { 2 - 5 } & Privé & Public / Pro & Littéraire & Presse \\
\hline quand même & $361(57 \%)$ & $272(48 \%)$ & $7(3,60 \%)$ & $7(3,40 \%)$ \\
\hline autres emplois & 267 & 292 & 184 & 199 \\
\hline
\end{tabular}

Tableau 11 : Séparation de quand même et des autres emplois adverbiaux

\subsubsection{La locution quand même}

À l'oral, cette locution adverbiale quand même correspond à 53\% des emplois invariables pour seulement 3,5\% à l'écrit. Nous ne ferons pas une étude approfondie de cette locution mais nous indiquerons quelques faits que les corpus permettent d'observer.

Dans les productions étudiées, cette locution s’interprète essentiellement comme un marqueur «facilitant la mise en acceptabilité d'une contradiction », pour reprendre les termes de Moeschler et Spengler (1981), ou pour «atténuer une proposition qui peut paraitre trop forte » (Beeching, 2005).

Dans cet emploi, quand même va pouvoir s’insérer dans des emplacements divers (Tableau 12) :

\begin{tabular}{|l|c|c|c|c|}
\hline & Début de la CV & $\begin{array}{c}\text { A l'intérieur de la } \\
\text { CV }\end{array}$ & Fin de la CV & Autres contextes \\
\hline Oral privé [255] & 22 & $131(51 \%)$ & 83 & 19 \\
\hline Oral pro [256] & 12 & $160(62 \%)$ & 44 & 40 \\
\hline
\end{tabular}

Tableau 12 : Place occupée par quand même dans les corpus oraux

\section{a) Début de la construction verbale :}

25a. voilà + et puis bon peut-être quand même pour moi alors ça c'est une indication pour moi (oral Pro)

En fait, quand même est rarement en position initiale, mais suit fréquemment un autre introducteur (mais, bon, voilà, etc.), ce qui laisse penser que, dans l'usage, la valeur d'atténuation exprimée par cette locution vient en renfort d'un autre terme :

25b. mais + quand même la plupart du temps on se dit moi je fais ce que je veux comme je veux (oral Pro) 
25c. voilà mais bon quand même il y a des inconvénients (oral Privé)

\section{b) À l'intérieur de la construction verbale :}

C'est la position majoritairement observée dans nos données :

26a. je pense quand même à la mort (oral Privé)

26b. je crois que j'ai quand même très souvent un a priori (oral Pro)

La locution se rencontre assez régulièrement entre l'auxiliaire et le participe et quelquefois aussi entre le sujet et le verbe :

27a. j’avais quand même fait un essai (oral Privé)

27b. sachant que le daim quand même blanchit (oral Privé)

c) Fin de la construction verbale :

28a. mais il y a eu quatre ans d'apprentissage quand même (oral Privé)

28b. allez + il fait pas froid + on est mieux en Martinique quand même hein (oral Pro)

\section{d) Autres contextes :}

29a. je suis dans un cadre quand même bien précis (oral Privé)

29b. puisque le propre quand même de l'image en bande dessinée c'est (oral Pro)

Cette locution se rencontre parfois lors de l'ajustement d'une formulation, quand même est réalisé immédiatement avant une reformulation :

30a.il y a quand même + il y a une espèce d'amalgame hein au niveau de cette jeunesse (oral Pro)

30b. mais + bon il y a quand même il y a un chemin qui s'est fait (oral Pro)

Pour conclure sur cette locution quand même, celle-ci est bien spécifique de l'oral, et comme le signale Beeching (2005, 2007), elle est utilisée par tous les locuteurs, quelle que soit la situation de parole et semble stable (tout autant produite en 1968 qu'aujourd'hui).

Dans les manuels, notamment de FLE, des équivalents moins marqués comme relevant de l'oral sont souvent proposés; c'est le cas pour tout de même, et dans un registre plus littéraire, pour néanmoins et cependant.

À noter cependant que ces termes ont souvent des possibilités distributionnelles moins développées, par exemple : cependant est peu envisageable en fin de construction, etc. Le choix de ces substituts, en position initiale, est assez conforme à ce qu'indiquent nos corpus (Tableau 13).

\begin{tabular}{|l|c|c|c|c|}
\hline \multirow{2}{*}{} & \multicolumn{2}{|c|}{ ORAL } & \multicolumn{2}{c|}{ ECRIT } \\
\cline { 2 - 5 } & Privé & Public / Pro & Littéraire & Presse \\
\hline cependant & 3 & 2 & 47 & 22 \\
\hline néanmoins & 2 & 5 & 10 & 18 \\
\hline tout de même & 4 & 8 & 5 & 9 \\
\hline
\end{tabular}

Tableau 13 : Les alternatives à quand même dans les corpus 
Il existe un emploi de cette locution quand bien même qui peut exprimer la concession adversative, comme dans l'exemple suivant produit à l'oral, par un locuteur professionnel (il s'agit en l'occurrence d'un avocat qui en fait le pivot d'une liste) :

(31) c'est la stricte vérité elle est très simple extrêmement délicate quand bien même je serais avocat quand bien même je parlerais bien quand bien même j'aurais les moyens de me défendre parce que l'on va me dire + peut-être vrai peut-être faux (oral Public)

C'est toutefois le seul exemple de cette locution qui figure dans nos données. On ne peut alors qu'être dubitatif quant à la place importante qu'occupe cette tournure dans les grammaires et au décalage que cela illustre entre l'usage et les descriptions de la langue proposées.

\subsubsection{Même adverbe modalisateur}

Dans cet emploi adverbial, la forme même est traditionnellement analysée comme un outil d'enchérissement (ou de gradation), (cf. Bat-Zeev, 2001 ; Mehdaoui, 2000), qui va pouvoir porter sur des éléments grammaticaux très différents : infinitifs (32), participes passés (33), ou autres formes :

32. les machineurs devaient même doubler la vitesse (écrit Litt)

33. j’ai même été assez loin (oral Pro)

Voici quelques emplois intéressants à commenter :

\section{a) Même suivi d'une préposition :}

La forme invariable même peut se réaliser avant une préposition comme dans :

34. Tout est légèreté, même dans l'agression (écrit Presse)

À l'oral, la présence de même s'accompagne fréquemment d'une antéposition de la séquence prépositionnelle régie. On peut considérer que cette forme devient alors un marqueur de focalisation qui signale la valeur informative du constituant placé avant le verbe :

35a. même pour mes petits fils je dois faire ça (oral Privé)

35b. même à un Bordelais je le dis (oral Privé)

35c. dans le métier de fleuriste même au bout de $\mathbf{1 0}$ ans on n'a pas tout appris (oral Privé)

À l'écrit, ce procédé est bien moins habituel mais pas absent :

36. D'ailleurs, même en Australie, on se met à fabriquer des 'fromages traditionnels' (écrit Presse)

\section{b) Même suivi de la particule négative pas (même pas) :}

La séquence même pas à l'oral est fréquente (près de 12\% des occurrences, 2 fois plus que dans les autres types de corpus) :

37. ça fait même pas deux ans (oral Privé) 
Cette modalisation de la particule négative permet de créer ce que l'on peut appeler un noyau non verbal (cf. Blanche-Benveniste, 1990, 2010), comme dans les exemples suivants :

38a. même pas en rêve (Bachelot entendu à la TV)

38b. même pas peur

\section{c) Même précédé de la particule négative pas (pas même) :}

L’adverbe même peut être précédé de pas, comme dans les exemples suivants :

39. Personne, pas même Mme Grandet, n'avait la permission de venir. (écrit, Litt)

40. et rien ne pouvait l'en empêcher - pas même elle. (écrit, Litt)

Dans ce contexte, la forme pas est très proche de ni. Il est alors intéressant de noter que cet emploi semble être spécifique de l'écrit Littéraire, où il représente près de $11 \%$ des occurrences. En revanche, on n'en relève pas à l'oral et très peu dans la Presse. En effet, l'oral semblant plutôt privilégier l'ordre inverse, même pas plutôt que pas même :

41. je me rappelle de rien même pas où j’étais (oral Privé)

\section{d) Même suivi d'une conjonction :}

On rencontre des utilisations de même en renforcement de certaines conjonctions : si, que et quand (Grevisse, 1980) semble considérer que la liste peut être étendue, il cite (\$ 1076) même avant que, suivi de points de suspension). Dans nos corpus, seules trois associations sont attestées (Tableau 14) :

\begin{tabular}{|l|c|c|c|c|}
\hline \multirow{2}{*}{} & \multicolumn{2}{|c|}{ ORAL } & \multicolumn{2}{c|}{ ECRIT } \\
\cline { 2 - 5 } & Privé & Public / Pro & Littéraire & Presse \\
\hline même si & 33 & 39 & 2 & 48 \\
\hline même que & 5 & 1 & 0 & 7 \\
\hline même quand & 2 & 5 & 2 & 0 \\
\hline
\end{tabular}

Tableau 14 : Même suivi d'une conjonction

On peut remarquer que les « conjonctions » renforcées par même n'introduisent pas toujours de réelles subordonnées (c'est-à-dire des constructions régies) mais parfois aussi des séquences associées, qui ne peuvent pas, par exemple, être extraites entre c'est... que.

\section{e) même si :}

Ces constructions semblent plutôt fonctionner comme des parenthèses (ce que la ponctuation souligne à l'écrit) :

42a. et et puis même si on accepte de les changer c'est très dur que le changement devienne pérenne (oral Pro)

42b. * c'est même si on accepte de changer que c'est très dur que le changement devienne pérenne 
43. Et pourtant, d'aller au château des Carpathes, même si l'on rémunérait son déplacement, cela ne pouvait lui convenir en aucune façon. (écrit Litt.)

L'oral mais aussi l'écrit de Presse favorise la locution même si (près de $24 \%$ des occurrences pour la Presse) qui est quasi absente de l'écrit Littéraire.

\section{f) même quand :}

On peut fournir des commentaires comparables pour même quand:

44a. eh bien écoutez je n'aliène pas ma liberté même quand on me dit la main sur le cœur que j'en garderais tous les avantages (oral HP)

44b. * c'est même quand on me dit que j'en garderais tous les avantages que je n'aliène pas ma liberté

45. Avec cet instinct, cette finesse de la femme qui a de l'esprit en toute chose, même quand elle console, Eugénie voulait tromper la douleur de son cousin en l'occupant de lui-même. (écrit Litt.)

On peut trouver, à l'oral, des suites susceptibles d'être ambiguës, comme dans (46) où quand peut s'interpréter comme une anticipation du second quand temporel ou comme la locution quand même suivie d'un quand temporel :

46. il faut réfléchir quand même quand on fait un bouquet (oral Privé)

il faut réfléchir

$$
\text { même quand on fait un bouquet }
$$

il faut réfléchir quand même quand on fait un bouquet

\section{g) même que :}

Nous n'avons relevé aucun exemple où même que s'interprète comme formant un seul bloc, comme dans les exemples du type :

47. il a été grossier même qu'il m’a traité de ... (cf. Le Goffic, 1993)

48. Même que le Colonel Maçon vient d'être tué (cf. TLFI)

Cette locution est pourtant souvent présentée comme typique de l'oral ou du langage familier ou "populaire ${ }^{11}$. La séquence même que est certes attestée dans nos corpus mais elle s'analyse différemment en deux items séparés, comme dans (49) :

49. il faut absolument construire l'Europe + je pense même que les changements nécessaires en France ne peuvent s'inscrire que dans une perspective européenne (oral Public)

Dans ces exemple, même s'insère entre je pense et sa valence complément réalisée sous la forme d'une que-P : que les changements....

11 Ball (2000) mentionne la forme même seulement dans ce type d'emploi, mais les exemples fournis (deux sur trois) sont issus d'un roman La dérobade de J. Cordelier (1976) et non de productions orales. 
5.3.3. Récapitulatif des différents emplois de même invariable (Tableau 15)

\begin{tabular}{|c|c|c|}
\hline \multicolumn{3}{|c|}{ même forme invariable } \\
\hline quand même & même modalisateur & même + conjonction \\
\hline $\begin{array}{l}\text { utilisation très importante } \\
\text { à l'oral, rare à l'écrit } \\
\text { placé plutôt à l'intérieur } \\
\text { de la construction } \\
\text { quand bien même: souvent } \\
\text { décrit, peu attesté }\end{array}$ & $\begin{array}{l}\text { devant une préposition : s'accompagne } \\
\text { à l'oral d'une antéposition fréquente du } \\
\text { Syntagme Prépositionnel (même à un } \\
\text { Bordelais je le dis) } \\
\text { pas même : dans l'écrit littéraire } \\
\text { même pas : à l'oral }\end{array}$ & $\begin{array}{l}\text { même si: absent de l'écrit } \\
\text { littéraire, bien présent à l'oral } \\
\text { et dans la presse } \\
\text { même quand: assez rare } \\
\text { même que: absent, doit souvent } \\
\text { être réanalysé }\end{array}$ \\
\hline
\end{tabular}

Tableau 15 : Les principaux emplois de même invariable

\subsection{Bilan}

Il nous a paru utile de détailler l'étude de cette forme même, tant elle illustre bien l'intérêt de travailler à partir de corpus diversifiés. Le résultat de ce travail n'est ni novateur ni complet, il permet toutefois de mieux apprécier les contextes les plus attestés. Cette analyse souligne parfois l'existence d'une discordance entre l'usage d'une forme et les développements qu'elle a suscités dans les grammaires ou les manuels, et elle a permis de mettre en lumière des associations parfois négligées.

$\mathrm{Au}$ terme de cette étude, on est tenté de suggérer que les différentes interprétations sémantiques (égalité/similitude, identité, modalité de renchérissement) et les différentes propriétés à la fois catégorielles et distributionnelles de cette forme devraient être hiérarchisées différemment dans les manuels. Des exemples comme (50)-(52) dans lesquels la forme même apparait plusieurs fois signalent bien la hiérarchie que l'on devrait opérer. L'adverbe de modalité avec effet de renchérissement est de toute évidence l'emploi majoritaire dans les productions contemporaines :
50. j'ai été moi-même une fois même invité par l'évêque d'Amiens (oral Privé)
51. il y a un état d'esprit commun quoi même si quand même j'imagine qu'il y a différents personnages oral (Privé)
52. parce que j’ai eu ce décalage quand même même en étant petite (oral Privé)

\section{Conclusion générale}

Le principal objectif de cet article était d'illustrer l'intérêt de fonder les descriptions grammaticales sur des corpus de nature et de « genres» différents. Pour certains points de syntaxe (comme les pronoms relatifs ou la succession des pronoms personnels), les analyses semblent peu susceptibles de modification, néanmoins l'appui sur des corpus, variés et équilibrés, s'avère utile puisqu'il permet d'apporter un éclairage nouveau, en faisant, par exemple, ressortir des écarts de distribution importants selon les formes étudiées et en mettant en lumière des associations lexicales fortes. La palette des observations sera d'autant plus riche que les descriptions 
s'appuieront effectivement sur différents types de corpus écrits ou oraux (Conrad \& Biber, 2009), comme le montre ici l'analyse plus détaillée de même. Le fait que l'on puisse aujourd'hui travailler sur un plus grand nombre d'usages offre de multiples pistes, que les manuels pourraient explorer avantageusement; cela leur permettrait de donner une représentation de la langue plus conforme à la réalité, du moins qui tiendrait compte un peu plus de son hétérogénéité.

\section{Bibliographie}

Abry, D. \& Chalaron, M-L. (2000), La grammaire des premiers temps (Volumes 1 \& 2), Grenoble, PUG.

ARNAVIELLE, T. \& G. SioufFi (2012), «Ecrire une grammaire du français aujourd'hui », Langue française, 176, p. 3-10.

ARrivÉ, M., GADET, F., GALMICHE, M. (1986), La grammaire d'aujourd'bui, Paris, Flammarion.

BALL, R. (2000), Colloquial French Grammar: A practical Guide, Oxford, Blackwell.

BAT-ZeEv SHYLDKRot, H. (2001), «Grammaticalisation et valeur sémantique : à propos d'un emploi particulier de même», C. Buridant, G. Kleiber \& J.-C. Pellat (éds.), Itinéraires linguistiques et grammaticaux, Louvain/Paris, Peeters, p. 1-13.

Baude, O. \& Dugua, C. (2011), «(Re)faire le corpus d'Orléans quarante ans après : quoi de neuf, linguiste ?», Corpus, 10, p. 99-118.

BeECHING, K. (2005), « Politeness-induced semantic change: the case of quand même », Language Variation and Change, 17, p. 155-186.

BEECHING, K. (2007), « La co-variation des marqueurs discursifs bon, c'est-à-dire, enfin, hein, quand même, quoi et si vous voulez: une question d'identité ? ", Langue française, 154, p. 78-93.

BÉrard, E. \& LAVEnNe, C. (1991), Modes d'emploi. Grammaire utile du français, Paris, Hatier/Didier.

Biber, D., JohAnsson, S., LeECH, G., CONRAD, S. \& FinegAn, E. (1999), Longman Grammar of Spoken and Written English, Londres/New York, Longman.

BILGER, M. (1998), «Le statut micro et macro-syntaxique de ET», dans M. Bilger, F. Gadet \& K. van den Eynde (éds), Analyse linguistique et approches de l'oral. Hommage à Claire Blanche-Benveniste, Louvain/Paris, Peeters, p. 91-102.

BILGER, M. (2000), Corpus : méthodologie et applications linguistiques, Paris, Honoré Champion.

BILGER, M. (2008), « De l'intérêt des corpus diversifiés pour les descriptions en (morpho)syntaxe. Réflexions et illustration avec le pronom relatif lequel», Verbum, 30/4, p. 275-286.

Bilger, M. \& CAPPEAU, P. (2004), «L'oral ou la multiplication des styles », Langage et société, 109 , p. $13-30$

BlanCHE-BenVeniste, C. (2010), Le français. Usages de la langue parlée. Louvain/Paris Peeters. 
Bonnard, H. (1981), Code du français courant, Paris, Magnard.

Branca-Rosoff, S, Fleury, S, Lefeuvre, F. \& Pires, M. (2011), «Constitution et exploitation d'un corpus de français parlé parisien », Corpus, 10, p. 81-98.

CADET, L. \& GUERIN, E. (éds.) (2012), «FLM, FLE, FLS au-delà des catégories », Le français aujourd'bui, 176.

CHAmbers, A. (2009), «Les corpus oraux en francais langue étrangère: authenticité et pédagogie », Mélanges CRAPEL, 31, p. 16-33.

CONRAD, S., \& BiBer, D. (2009), Real Grammar: A Corpus-Based Approach to English, New York, Pearson.

Delatour, Y., Jennepin, D., LÉOn-Dufour, M. \& Teyssier B. (2004), Nouvelle grammaire du français, Paris Hachette.

DELIC (équipe) (2004), « Présentation du corpus de référence du français parlé », Recherches sur le français parlé, 18, p. 11-42.

DE SALINS, G.-D. (1996), Grammaire pour l'enseignement/apprentissage du FLE, Paris, Didier/ Hatier.

Detey, S., Durand, J., LAKs, B. \& LyCHe, C., (éds) (2010), Les variétés du français parlé dans l'espace francophone. Ressources pour l'enseignement, Paris, Ophrys.

Flaux, N. (2008), «Les pronoms indéfinis en français : une classe à (re)définir», Travaux de linguistique, 56, p. 7-46.

Gadet, F., Ludwig, R. \& PFÄNDER, S. (2009) : «Francophonie et typologie des situations », Cabiers de linguistique, 34/1, p. 143-162.

Grevisse, M. (1980), Le bon usage, Paris-Gembloux, Duculot.

HABERT, B. (2000), «Des corpus représentatifs : de quoi, pour quoi, comment?», dans M. Bilger (éd.), Linguistique sur corpus : études et réflexions, Perpignan, Presses Universitaires de Perpignan, p. 11-58.

Halliday, M. A. K. (1991), "Corpus studies and probabilistic grammar», dans K. Aijmer \& B. Altemberg (éds.), English Corpus Linguistics: Studies in Honour of Jan Svartvik, Londres/ New-York, Longman, p. 30-43.

Job, B., Mis, B. \& Pissavy, A-M. (1986), Comment dire? Grammaire simplifiée, Paris, Clé International.

LAFFON, M. \& ZEGGAH-WUYTS, F. (2009), La grammaire en action, Paris, Clé International.

LE GOFFIC, P. (1993), Grammaire de la langue française, Paris, Hachette Education.

MCBRIDE, N. (1997), Grammaire française, Paris, Hachette.

MARTin, R., (1975), "Sur l'unité du mot même », Travaux de linguistique et de littérature, 13/1, p. 227-243.

MCCARThy, M. (2004), Toucbstone: From Corpus to Coursebook, Cambridge, Cambridge University Press.

MeHdaOuI, M. (2000), L'adverbe même en français contemporain : concession et/ou enchérissement, thèse de doctorat, Strasbourg. 
Comment les données de corpus pourraient renouveler les manuels de grammaire?

Moeschler, J. \& N. De Spengler (1981), "Quand même: de la concession à la réfutation », Cabiers de Linguistique française, 2, p. 53-112.

Miller, J. \& WeINERT, R. (1998), Spontaneous Spoken Language. Syntax and Discourse, Oxford, Oxford University Press.

RAstier, F. (2005), «Enjeux épistémologiques de la linguistique de corpus » dans G. Williams (éd.), La linguistique de corpus, Rennes, Presses Universitaires de Rennes, p. 31-45.

Riegel, M., Pellat, J.-C. \& Rioul, R. (2009), Grammaire méthodique du français, Paris, Presses Universitaires de France.

SinClair, J. (1991), Corpus, Concordance, Collocation, Oxford, Oxford University Press.

Tognini-Boneldi, E. (2001), Corpus Linguistics at Work, Amsterdam, John Benjamins.

Van Peteghem, M. (1997), "Sur un indéfini marginal : même exprimant l’identité », Langue française, 116, p. 61-80.

Véronique, D., Carlo, C., Granget, C., KIM, J-O. (2009), L'acquisition de la grammaire du français, langue étrangère, Paris, Didier. 